\title{
Body roundness index is a superior indicator to associate with the cardio-metabolic risk: evidence from a cross-sectional study with 17,000 Eastern-China adults
}

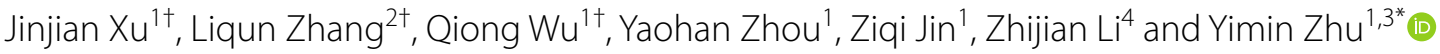

\begin{abstract}
Background: To investigate the ability of body shape index (ABSI), body roundness index (BRI), waist circumference (WC), body mass index (BMI), waist-to-hip ratio (WHR), waist-to-height ratio (WHtR), and body adiposity index (BAl) for predicting non-adipose cardio-metabolic risk.

Methods: A total of 17,360 Chinese subjects aged 18-95 years old who escaped cardiovascular disease (CVD) or diabetes were recruited in the cross-sectional study. Anthropometric and biochemical parameters were assessed. Receiver operating characteristic curve (ROC) and multinomial logistic regression were conducted to examine the association of anthropometric indicators with cardio-metabolic risk factors.

Results: The mean age of subjects were $53.7(13.1)$ years, $41.6 \%$ were males. The areas under the curve (AUC) demonstrated that WC, BMI, WHR, WHtR and BRI were able to predict high cardio-metabolic risk (AUC > 0.70). Meanwhile, multinomial logistic regression showed BRI was significantly associated with high cardio-metabolic risk (OR 3.27, 95\% Cl 3.01-3.55). The optimal cut-off values of BRI for high cardio-metabolic risk were ( $<60 \mathrm{y}: 3.49 \mathrm{vs}$. $\geq 60 \mathrm{y}: 3.46)$ in males and (<60 y: 3.47 vs. $\geq 60 \mathrm{y}: 3.60)$ in females.

Conclusions: WC, BMI WHR, and WHtR were potential obesity indicators in discriminating high cardio-metabolic risk, while BAI or ABSI was not. Moreover, BRI revealed superior predictive capacity and significant association with accumulated cardio-metabolic risk factors.
\end{abstract}

Keywords: Body roundness index, Cardio-metabolic risk factors, Eastern-China adults

\section{Background}

Metabolic syndrome (MetSy) is a serious metabolic disorder consisting of obesity, hypertension, abnormal metabolism of lipoprotein and plasma glucose, which plays a vital role in the development of atherosclerotic heart disease, stroke and diabetes mellitus [1-3]. So far,

\footnotetext{
*Correspondence: zhuym@zju.edu.cn

${ }^{\dagger}$ Jinjian Xu, Liqun Zhang and Qiong Wu have contributed equally

${ }^{1}$ Department of Epidemiology and Biostatistics, School of Public Health,

Zhejiang University, Hangzhou 310058, Zhejiang, China

Full list of author information is available at the end of the article
}

over $33.9 \%$ of older adults reached abnormal metabolism in China and caused a large disease-related healthcare burden [4-7].

MetSy was considered as an intermediate trait in the progression of severe cardiovascular disease (CVD) $[8,9]$ and can contribute to the risk of diabetes[10, 11]. However, obesity is an important component of metabolic syndrome that can independently contribute to the remaining components $[12,13]$. Meanwhile, epidemiological studies have shown that obesity itself was a vital risk factor for hypertension [14], and cardiovascular 
disease [15] and diabetes [16]. Thus, that is necessary to explore the independent association between obesity and risk of CVD. Moreover, the remaining components of MetSy including hypertension, elevated fasting glucose (FPG), elevated triglycerides (TG), and reduced highdensity lipoprotein cholesterol (HDL-C) were studied as the risks of CVD in previous studies [17-19]. Thus, the residual components of MetSy that excluding obesity were clustered as non-adipose cardio-metabolic risk factors will be more suitable for investigating the progress of cardiovascular disease or other adverse traits $[4,20]$. Moreover, serum uric acid (SUA) is the end product of nucleic acid purine metabolism in body [21-24]. Previous studies revealed that an alteration of SUA level was associated with abnormal glucose and hyperuricemia, and it was considered to be a risk factor for MetSy or metabolic disorders [25-27].

Obesity was mainly due to the disproportionate growth of adipose tissue and lean body mass which can lead to further morbidity and mortality from cardiovascular disease [28, 29]. Recently, obesity was increasing rapidly worldwide and reached epidemic levels in China, which poses heavy public health and economic burdens [30]. The scanning of dual-energy X-ray absorptiometry (DXA), hydrostatic weighing, bioelectrical impedance and even skinfold thickness were considered as accurate methods for evaluating fat mass and distribution, whereas the use of such measurement was limited due to its complexity and/or cost [31, 32]. Epidemiological studies have revealed the anthropometric measures were acceptable indicators in evaluating obesity status for their simplicity and usefulness [20,33-35]. The body mass index (BMI) has been considered as a diagnostic index for general obesity and could reflect the overall distribution of body fat [36, 37]. Meanwhile, waist circumference (WC), body adiposity index (BAI), waist-tohip ratio (WHR) and waist-to-height ratio (WHtR) were studied to predict metabolic risk in previous studies [5, 38-40]. However, the traditional anthropometric indices failed to discriminate between fat and muscle mass [41, 42]. Recently, body shape index (ABSI) which reflects the body shape using waist circumference (WC), weight, and height and body roundness index (BRI) combines height and WC to predict the percentage of total and regional fat have been developed to overcome the limits of traditional obesity markers [33, 43-49]. A previous study found the two new indices were associated with abdominal adipose and more associated with cardiometabolic risk, onset of diabetes and premature mortality hazards than BMI and WC $[4,50,51]$. However, few shreds of evidence compared ABSI, BRI with common anthropometric indicators such as WC in the assessment of cardiometabolic risk factors in Chinese.
In this study, we clustered elevated blood pressure (BP), elevated FPG, elevated TG, reduced HDL-C and elevated serum uric acid (SUA) as non-adipose cardio-metabolic risk factors and adopted seven anthropometric indices including waist circumference (WC), body mass index (BMI), waist-to-hip ratio (WHR), waist-to-height ratio (WHtR), body adiposity index (BAI), body shape index (ABSI) and body roundness index (BRI) to conduct a population-based cross-sectional study to assess the ability of obesity indicators for predicting non-adipose cardio-metabolic risk and explore the pre-cardiovascular status in Chinese adults.

\section{Methods \\ Study population}

The cross-sectional data sources were the baseline records from 2009 to 2012 in Zhejiang Metabolic Syndrome Cohort (ZJMSC). ZJMSC is an ongoing community-based longitudinal study initiated in 2009 years included four communities and consisted of 22,649 participants aged 18-75 years old who have lived in Zhejiang province (Hangzhou, Zhoushan, Jiaxing, Jinhua) for more than 5 years and the follow-up visit was held from 2009 to 2019. A total of 22,649 participants (9,527 males and 13,122 females, mean age were $54.86 \pm 14.2$ years old) were recruited in the present study. Subjects were defined as the young group ( $<60$ years old) and the older group ( $\geq 60$ years old). Exclusion criteria: (1) severe infective diseases and malignancies in baseline; (2) patients with type 2 diabetes mellitus (T2DM), cardiovascular disease (CVD) and stroke in baseline; (3) less than 18 years old; (4) without biochemical and anthropometric data. A total of 17,360 (7,226 males and 10,134 females) participants met these criteria and were finally included in this study. Additionally, inclusion criteria and selection flowchart for all samples as shown in Fig. 1. This study was approved by the Human Research Ethics Committee of Zhejiang University, Zhejiang, China. All participants provided written informed consent before participation.

\section{Measurements \\ Anthropometric variables and covariates}

For each participant, covariates of demographic characteristics, smoking and alcohol drinking status, diet, and physical activity was collected with questionnaire-based interview. In the present study, smoking and drinking were categorized as current, former and never. Current smokers and drinkers were defined as those who were still smoking or drinking during the investigation. Former smokers and drinkers were those who had a history of smoking or drinking and stopped for at least one year. The occupational labor strength was defined as mild labor, moderate labor, and heavy labor. 


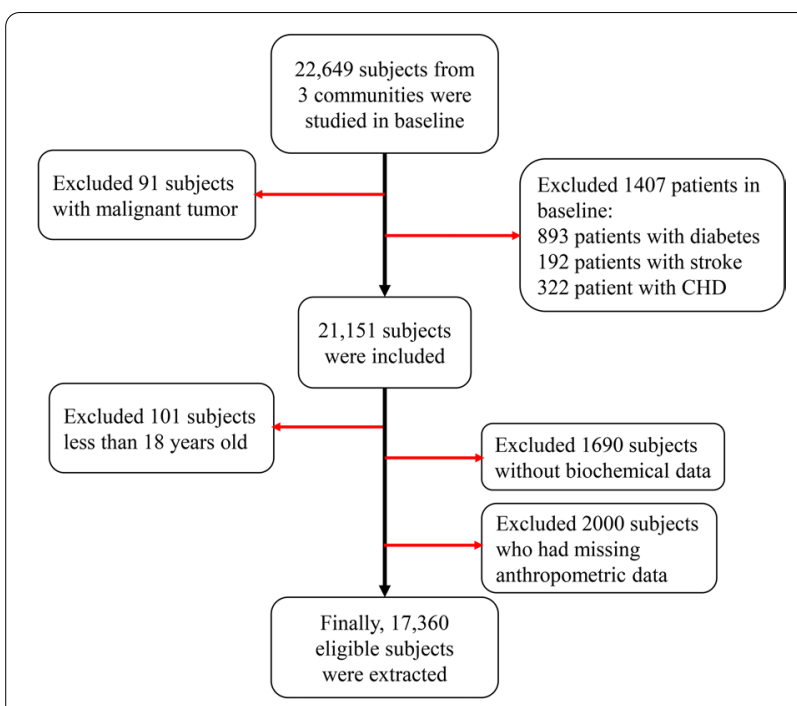

Fig. 1 Flowchart of inclusion criteria for participants in this study

Height $(\mathrm{cm})$, body weight $(\mathrm{kg})$, waist circumference (WC; $\mathrm{cm}$ ), hip circumference (HC; $\mathrm{cm})$, systolic blood pressure (SBP) and diastolic blood pressure (DBP) were measured by trained assistants using medical equipment. Height and weight were measured with the subjects wearing light clothing and no shoes. Height was recorded to the nearest $0.1 \mathrm{~cm}$ and body weight to the nearest $0.1 \mathrm{~kg}$. Body mass index (BMI) was computed as weight $(\mathrm{kg})$ divided by height squared $\left(\mathrm{m}^{2}\right)$. The girth of the midpoint between the lowest point of the rib and the upper edge of the iliac crest were calculated as waist circumference (WC). The length of the horizontal position of the hip protrusion was calculated as the hip circumference (HC) [52]. The measurements of $\mathrm{WC}$ and $\mathrm{HC}$ were taken to the nearest $0.1 \mathrm{~cm}$. Waist-to-hip ratio (WHR) and waist-to-height ratio (WHtR) were respectively calculated as WC divided by HC and WC divided by height. BAI was calculated as $\mathrm{HC}(\mathrm{cm}) /$ height $^{1.5}(\mathrm{~m})$ minus 18 [53]. ABSI was calculated using the formula as $\mathrm{ABSI}=\frac{\mathrm{WC}}{\mathrm{BMI}^{2 / 3} \text { Height }^{1 / 2}}$ and BRI was calculated using the formula as BRI $=364.2-365.5 \times \sqrt{1-\left(\frac{(\mathrm{WC} /(2 \pi))^{2}}{(0.5 \text { Height })^{2}}\right)} . \quad$ Sitting blood pressure was measured 2 times after at least 10 minutes of rest using the standardized desktop sphygmomanometer. The average blood pressure derived from two measurement readings was used $[43,51]$. All variables were collected according to standard interview guidelines and standard protocols [54-57].

\section{Biochemical measurements}

After an overnight fast of at least 12 hours, intravenous blood samples of each participant were collected, with one tube for Ethylene Diamine Tetraacetic Acid (EDTA) anticoagulation and one tube for separation gel coagulation, then centrifuged at $4000 \mathrm{rpm}$ for $15 \mathrm{~min}$ at $4^{\circ} \mathrm{C}$, the aliquots were stored at $-80^{\circ} \mathrm{C}$ until use. Fasting plasma glucose (FPG) was measured by the hexokinase method, SUA and lipid profile containing total cholesterol (TC), triglycerides (TG), high-density lipoprotein cholesterol (HDL-C) and low-density lipoprotein cholesterol (LDLC) were measured by the enzymatic method using an Abbott Aeroset autoanalyzer. Alanine transaminase (ALT) and aspartate transaminase (AST) were measured with a rating method. Blood urea nitrogen was measured with uricase ultraviolet method and serum creatinine with a picric acid method.

\section{Criteria for cardio-metabolic risk factors}

According to the diagnosis criteria of MetSy by the International Diabetes Federation (IDF) in 2009 [58], the comprehensive non-adipose cardiometabolic risk factors included the following items: (1) elevated BP: systolic blood pressure (SBP) ( $\geq 130 \mathrm{mmHg}$ ) or diastolic blood pressure (DBP) ( $\geq 85 \mathrm{~mm} \mathrm{Hg}$ ), or ongoing antihypertensive medications; (2) elevated FPG: FPG $\geq 5.6 \mathrm{mmol} / \mathrm{L}$ and/or diagnosis of type 2 diabetes, or ongoing antidiabetic treatment; (3) elevated TG: TG $\geq 1.7 \mathrm{mmol} / \mathrm{L}$; (4) reduced HDL-C: HDL-C $<1.03 \mathrm{mmol} / \mathrm{L}$ in men and $\mathrm{HDL}-\mathrm{C}<1.29 \mathrm{mmol} / \mathrm{L}$ in women. In addition, we also enrolled: (5) elevated SUA: SUA $>420 \mu \mathrm{mol} / \mathrm{L}$ according to the diagnosis criteria of hyperuricemia in 2000 [59]. The people with three or more risks were diagnosed as "high cardio-metabolic risk population" in present study.

\section{Statistics}

The continuous variables were presented as means and standard deviations (SD), categorical variables as counts or percentages. The one-way analysis of variance and the chi-squared test were used to compare the baseline characteristics of participants stratified by the number of risk factors. The linear correlation was conducted to explore the relationship among multiple anthropometric indicators and revealed the mutative trend between median of anthropometric indicators and the number of cardiometabolic risk factors. In addition, multiple comparisons were conducted between the highest risk group and residual groups. Adjusted multinomial logistic regression was conducted to examine the associations of adiposity indicators with cardio-metabolic risk. To determine the optimal cut-off values and to compare the ability of the obesity indices for predicting multiple metabolic risk factors, the receiver operating 
characteristic curve (ROC) and area under the curve (AUC) were performed and optimal cut-off values were identified from the maximum Youden index (sensitivity plus specificity-1). The Hanley and McNeil method was used to compare the inter-group differences of AUCs. The analysis was performed by SPSS 25.0 software. All analyses were two-sided, and the difference was statistically significant at $P<0.05$.

\section{Results}

\section{Basic characteristics of the objects}

According to the number of cardio-metabolic risk factors, subjects were divided into non-metabolic risk group, low metabolic risk group $(<3)$ and high metabolic risk group $(\geq 3)$, and the differences of basic characteristics were analyzed by the presence of cardio-metabolic risk factors (Table 1). The proportion of females was higher in high metabolic risk groups (58.7\%). Participants with high cardio-metabolic risk were older than those with none or low metabolic risk $(P<0.001)$. In addition, participants with high cardiometabolic risk were more likely to have higher values of SBP, DBP, FPG, TG, HDL-C, LDL-C, and SUA and lower levels of HDL-C than the other groups $(P<0.001)$. Moreover, participants with high cardio-metabolic risk were more likely to have higher BMI, WC, HC, WHR, WHtR, BAI, ABSI and BRI $(P<0.001)$. In addition, the average values of SBP, FPG and TG were abnormal in high cardio-metabolic risk group, which were 146.58 (19.23) $\mathrm{mmHg}, 5.58$ (1.52) $\mathrm{mmol} / \mathrm{L}$ and 2.91 (2.04) $\mathrm{mmol} / \mathrm{L}$, respectively.

\section{Cardio-metabolic risk factors and anthropometric indicators}

Table 2 shows the mean value of anthropometric parameters in multiple groups by the presence of cardio-metabolic factors. WC, BMI, WHR, WHtR, BAI, ABSI and BRI increased significantly with the number of cardiometabolic risk factors $\left(P_{-}\right.$trend $\left.<0.01\right)$. Moreover, the averages of indicators in the highest cardio-metabolic risk group were greater than residual groups $(P<0.01)$, and the values of WC, BMI, WHR, WHtR, BAI, ABSI and BRI were $87.25 \mathrm{~cm}, 26.68 \mathrm{~kg} / \mathrm{m}^{2}, 0.94,0.54,26.98$, 0.078 and 4.23 in males, in females were $88.69 \mathrm{~cm}, 26.04$ $\mathrm{kg} / \mathrm{m}^{2}, 0.92,0.58,32.31,0.081$ and 4.97 . Meanwhile, the correlation matrix between anthropometric indicators was presented in Additional file 1: Table S1. In both men and women, BRI showed a strong correlation with WC (men: $\mathrm{r}=0.93, P<0.01$; women: $\mathrm{r}=0.94, P<0.01$ ) and WHtR (men: $\mathrm{r}=0.99, P<0.01$; women: $\mathrm{r}=0.99, P<0.01$ ), while the linear correlation between BRI and BMI (men: $\mathrm{r}=0.80, P<0.01$; women: $\mathrm{r}=0.77, P<0.01)$ was relatively weak (Additional file 1: Table S1, Additional file 2: Additional Fig. S1a, b).

\section{AUCs of anthropometric indicators for cardio-metabolic risk factors}

Based on the result of ROC, the AUCs for each anthropometric indicator to predict multiple cardio-metabolic risk factors were presented in Additional file 1: Table S2. The AUCs presented that WC, BMI, WHR, WHtR, and BRI had better predictive ability for elevated TG in males and their AUCs were 0.70, 0.70, 0.68, 0.70 and 0.70. Similarly, the superior abilities of WC, BMI, WHR, WHtR and BRI to predict TG were observed in female, the AUCs were $0.69,0.68,0.67,0.69$ and 0.69 . In addition, WC, WHtR and BRI displayed a superior predictive ability to high SUA in females, with AUCs of 0.69, 0.70 and 0.70, respectively. Moreover, the AUCs of obesity parameters for predicting cardio-metabolic risk factors were significant (Additional file 1: Table S2, Additional file 3: Fig. S2a-j).

Table 3 shows that WC, BMI, WHR, WHtR and BRI revealed similar abilities to predict high cardio-metabolic risk. In the young group ( $<60$ years), the AUCs for males were $0.74,0.74,0.73,0.73,0.73$, for females were 0.73 , $0.73,0.71,0.73$ and 0.73 . However, in older group $(\geq 60$ years), the AUCs of WC, BMI, WHR, WHtR and BRI were $0.73,0.73,0.70,0.73$ and 0.73 for males, and were $0.69,0.70,0.65,68$ and 0.68 for females. In addition, the best cut-off values of WC, BMI, WHR, WHtR and BRI were $84.45 \mathrm{~cm}, 24.04 \mathrm{~kg} / \mathrm{m}^{2}, 0.88,0.51$ and 3.49 in males within young group ( $<60$ years) and in older group $(\geq 60$ years), the best cut-off values were $84.75 \mathrm{~cm}, 23.24 \mathrm{~kg} /$ $\mathrm{m}^{2}, 0.90,0.51$ and 3.46. Meanwhile, in females, the best cut-off values of WC, BMI, WHR, WHtR and BRI were $78.90 \mathrm{~cm}, 22.94 \mathrm{~kg} / \mathrm{m}^{2}, 0.85,0.51$ and 3.47 within young group ( $<60$ years) and were $78.85 \mathrm{~cm}, 23.73 \mathrm{~kg} / \mathrm{m}^{2}, 0.88$, 0.51 and 3.60 in older group ( $\geq 60$ years). Meanwhile, the sensitivity, specificity and Youden index of anthropometric measurements to predict high cardio-metabolic risk was shown in Table 3. In addition, the differences of predictive ability for high cardio-metabolic risk factors by anthropometric indicators were significant $(\mathrm{P}<0.001)$ (Table 3; Fig. 2a-d).

\section{Associations of anthropometric indicators with cardio- metabolic risk factors}

The results of logistic regression revealed that WC, BMI, WHR, WHtR and BAI, ABSI and BRI were significantly associated with accumulated cardio-metabolic risk factors in model 1 (Table 4). After adjusted covariates, the association of ABSI with high cardio-metabolic risk was suppressed (OR 1.08, 95\% CI 1.01-1.16). 
Table 1 Baseline characteristics of study subjects

\begin{tabular}{|c|c|c|c|c|c|}
\hline \multirow[t]{2}{*}{ Variables } & \multirow[t]{2}{*}{$\mathbf{N}$} & \multicolumn{3}{|c|}{ Number of cardio-metabolic abnormalities } & \multirow[t]{2}{*}{$P$ value } \\
\hline & & None & $<3$ & $\geq 3$ & \\
\hline Sex, N (\%) & & & & & $<0.001$ \\
\hline Male & 7226 & $1785(38.3 \%)$ & $4308(43.3 \%)$ & $1133(41.3 \%)$ & \\
\hline Female & 10,134 & $2876(61.7 \%)$ & $5650(56.7 \%)$ & $1608(58.7)$ & \\
\hline Age (years) & 17,290 & $48.44(14.88)$ & $56.63(14.11)$ & $59.66(12.57)$ & $<0.001$ \\
\hline Smoker, N (\%) & 3575 & $923(21.21 \%)$ & $2099(22.35 \%)$ & $553(21.63 \%)$ & 0.295 \\
\hline Alcohol drinker, N (\%) & 4191 & $1098(26.94 \%)$ & $2438(27.66 \%)$ & $655(26.57 \%)$ & 0.471 \\
\hline Occupational labor strength, N (\%) & & & & & $<0.001$ \\
\hline Mild & 10,040 & $2444(57.93 \%)$ & $5726(62.73 \%)$ & $1870(72.12 \%)$ & \\
\hline Moderate & 3049 & $958(22.71 \%)$ & $1754(19.22 \%)$ & $337(13.00 \%)$ & \\
\hline Heavy & 2851 & $817(19.36 \%)$ & $1648(18.05 \%)$ & $386(14.89 \%)$ & \\
\hline Height (cm) & 17,057 & $160.69(7.95)$ & $160.05(8.33)$ & $159.8(8.57)$ & $<0.001$ \\
\hline Weight (kg) & 16,995 & $55.91(8.67)$ & $59.48(10.29)$ & $64.2(10.75)$ & $<0.001$ \\
\hline$W C(\mathrm{~cm})$ & 17,018 & $75.11(8.36)$ & $80.27(9.16)$ & $85.8(8.6)$ & $<0.001$ \\
\hline $\mathrm{HC}(\mathrm{cm})$ & 17,022 & $90.13(6.09)$ & $92.11(6.71)$ & $94.72(6.7)$ & $<0.001$ \\
\hline BMI $\left(\mathrm{kg} / \mathrm{m}^{2}\right)$ & 16,967 & $21.62(2.79)$ & $23.15(3.16)$ & $25.06(3.14)$ & $<0.001$ \\
\hline WHR & 17,004 & $0.83(0.07)$ & $0.87(0.07)$ & $0.91(0.06)$ & $<0.001$ \\
\hline WHtR & 16,961 & $0.47(0.05)$ & $0.5(0.06)$ & $0.54(0.05)$ & $<0.001$ \\
\hline BAl & 16,965 & $26.4(3.95)$ & $27.65(4.15)$ & $29.07(4.29)$ & $<0.001$ \\
\hline$A B S I$ & 16,878 & $0.08(0.005)$ & $0.08(0.005)$ & $0.08(0.005)$ & $<0.001$ \\
\hline BRI & 16,961 & $2.83(0.95)$ & $3.47(1.12)$ & $4.15(1.12)$ & $<0.001$ \\
\hline $\operatorname{ALT}(U / L)$ & 11,645 & $20.00(13.92)$ & $23.01(16.79)$ & $28.59(20.66)$ & $<0.001$ \\
\hline AST (U/L) & 9354 & $24.99(10.71)$ & $27.45(14.65)$ & $29.85(16.36)$ & $<0.001$ \\
\hline Serum creatinine (umol/L) & 9615 & $89.22(12.80)$ & $91.12(16.81)$ & $93.94(17.71)$ & $<0.001$ \\
\hline Blood urea nitrogen (mmol/L) & 9358 & $5.6(1.61)$ & $5.86(2.86)$ & $5.71(1.66)$ & $<0.001$ \\
\hline $\mathrm{SBP}(\mathrm{mmHg})$ & 17,359 & $112.52(10.2)$ & $133.94(21.15)$ & $146.58(19.23)$ & $<0.001$ \\
\hline $\mathrm{DBP}(\mathrm{mmHg})$ & 17,359 & $68.97(7.85)$ & $79.01(11.65)$ & $84.64(11.1)$ & $<0.001$ \\
\hline $\mathrm{FPG}(\mathrm{mmol} / \mathrm{L})$ & 17,360 & $4.6(0.48)$ & $4.93(1)$ & $5.58(1.52)$ & $<0.001$ \\
\hline $\mathrm{TG}(\mathrm{mmol} / \mathrm{L})$ & 17,360 & $0.97(0.32)$ & $1.52(1.04)$ & $2.91(2.04)$ & $<0.001$ \\
\hline $\mathrm{HDL}-\mathrm{C}(\mathrm{mmol} / \mathrm{L})$ & 17,360 & $1.62(0.33)$ & $1.5(4.4)$ & $1.16(0.31)$ & $<0.001$ \\
\hline $\mathrm{LDL}-\mathrm{C}(\mathrm{mmol} / \mathrm{L})$ & 17,344 & $2.52(0.73)$ & $2.76(0.79)$ & $2.88(0.84)$ & $<0.001$ \\
\hline SUA ( $\mu \mathrm{mol} / \mathrm{L})$ & 17,360 & $280.96(65.57)$ & $315.93(87.93)$ & $377.27(109.3)$ & $<0.001$ \\
\hline Elevated BP & 8578 & 0 & $6076(61.0 \%)$ & $2502(91.3 \%)$ & $<0.001$ \\
\hline Elevated FPG & 2574 & 0 & $1374(13.8 \%)$ & $1200(43.8 \%)$ & $<0.001$ \\
\hline Elevated TG & 5134 & 0 & $2793(28.0 \%)$ & $2341(85.4 \%)$ & $<0.001$ \\
\hline Reduced HDL-C & 4506 & 0 & 2651 (26.6\%) & 1855 (67.7\%) & $<0.001$ \\
\hline Elevated SUA & 2222 & 0 & $1196(12.0 \%)$ & 1026 (37.4\%) & $<0.001$ \\
\hline
\end{tabular}

Data are expressed as mean \pm standard deviation or counts (percentages)

WC waist circumference, $H C$ hip circumference, $B M I$ body mass index, WHR waist-to-hip ratio, WHtR waist-to-height ratio, BAl body adiposity index, $A B S /$ a body shape index, $B R I$ body roundness index, SBP systolic blood pressure, $D B P$ diastolic blood pressure, $F P G$ fasting plasma glucose, $T G$ triglyceride, $H D L-C$ high-density lipoprotein cholesterol, LDL-C low-density lipoprotein cholesterol, SUA serum uric acid, BP blood pressure, $A L T$ alanine transaminase, AST aspartate transaminase

Meanwhile, WC (OR 1.16, 95\% CI 1.15-1.17), BMI (OR 1.45, 95\% CI 1.41-1.47), WHR (OR 1.79, 95\% CI 1.74-1.83), WHtR (OR 1.76, 95\% CI 1.67-1.85) and BAI (OR 1.23, 95\% CI 1.21-1.26) were associated with high accumulated cardio-metabolic risk in model 3. Moreover, BRI demonstrated the strongest relationship to accumulated cardio-metabolic risk regardless of lifestyles (OR 3.27, 95\% CI 3.01-3.55). 
Table 2 The alteration of anthropometric indicators with the increase of cardiometabolic factors

\begin{tabular}{|c|c|c|c|c|c|c|c|}
\hline \multirow[t]{2}{*}{ Variables } & \multicolumn{7}{|c|}{ Number of cardiometabolic risk factors } \\
\hline & 0 & 1 & 2 & 3 & 4 & 5 & $P \_$trend \\
\hline Male & 1742 & 2400 & 1690 & 810 & 250 & 28 & \\
\hline WC & $77.17 \pm 7.94^{* *}$ & $80.52 \pm 8.48^{* *}$ & $83.73 \pm 8.91^{* *}$ & $87.45 \pm 8.48^{* *}$ & $89.15 \pm 7.42^{*}$ & $89.25 \pm 8.79$ & $<0.001$ \\
\hline BMl & $21.59 \pm 2.64^{* *}$ & $22.71 \pm 2.91^{* *}$ & $23.87 \pm 3.06^{* *}$ & $25.08 \pm 3.12^{* *}$ & $25.60 \pm 2.84^{* *}$ & $26.68 \pm 3.26$ & $<0.001$ \\
\hline WHR & $0.85 \pm 0.06^{* *}$ & $0.88 \pm 0.06^{* *}$ & $0.90 \pm 0.06^{* *}$ & $0.92 \pm 0.06^{* *}$ & $0.93 \pm 0.05^{* *}$ & $0.94 \pm 0.05$ & $<0.001$ \\
\hline $\mathrm{WHtR}$ & $0.46 \pm 0.05^{* *}$ & $0.48 \pm 0.05^{* *}$ & $0.50 \pm 0.05^{* *}$ & $0.52 \pm 0.05^{* *}$ & $0.54 \pm 0.05^{*}$ & $0.54 \pm 0.06$ & $<0.001$ \\
\hline $\mathrm{BAl}$ & $23.98 \pm 3.08^{* *}$ & $24.89 \pm 3.27^{* *}$ & $25.72 \pm 3.23^{* *}$ & $26.28 \pm 3.24^{*}$ & $26.75 \pm 3.47^{*}$ & $26.98 \pm 5.39$ & $<0.01$ \\
\hline$A B S I$ & $0.077 \pm 0.005^{* *}$ & $0.078 \pm 0.005^{*}$ & $0.079 \pm 0.005^{*}$ & $0.079 \pm 0.004^{* *}$ & $0.080 \pm 0.004^{* *}$ & $0.078 \pm 0.006$ & $<0.01$ \\
\hline BRI & $2.73 \pm 0.84^{* *}$ & $3.13 \pm 0.95^{* *}$ & $3.49 \pm 0.99^{* *}$ & $3.87 \pm 0.99^{* *}$ & $4.10 \pm 0.93^{* *}$ & $4.23 \pm 1.26$ & $<0.001$ \\
\hline Female & 2848 & 3216 & 2282 & 1228 & 306 & 28 & \\
\hline WC & $73.81 \pm 8.37^{* *}$ & $77.68 \pm 9.06^{* *}$ & $81.14 \pm 9.13^{* *}$ & $83.91 \pm 8.44^{* *}$ & $86.29 \pm 8.26^{* *}$ & $88.69 \pm 7.16$ & $<0.001$ \\
\hline $\mathrm{BMI}$ & $21.67 \pm 2.88^{* *}$ & $22.75 \pm 3.12^{* *}$ & $23.87 \pm 3.30^{* *}$ & $24.79 \pm 3.08^{* *}$ & $25.73 \pm 3.30^{* *}$ & $26.04 \pm 2.88$ & $<0.001$ \\
\hline WHR & $0.82 \pm 0.07^{* *}$ & $0.85 \pm 0.07^{* *}$ & $0.87 \pm 0.07^{* *}$ & $0.89 \pm 0.07^{* *}$ & $0.90 \pm 0.06^{* *}$ & $0.92 \pm 0.06$ & $<0.001$ \\
\hline $\mathrm{WHtR}$ & $0.47 \pm 0.06^{* *}$ & $0.50 \pm 0.06^{* *}$ & $0.52 \pm 0.06^{* *}$ & $0.54 \pm 0.06^{* *}$ & $0.56 \pm 0.06^{* *}$ & $0.58 \pm 0.06$ & $<0.001$ \\
\hline BAl & $27.92 \pm 3.65^{* *}$ & $29.08 \pm 3.67^{* *}$ & $30.03 \pm 3.89^{* *}$ & $30.67 \pm 3.77^{* *}$ & $31.60 \pm 3.90^{* *}$ & $32.31 \pm 4.79$ & $<0.01$ \\
\hline$A B S I$ & $0.076 \pm 0.006^{* *}$ & $0.078 \pm 0.006^{* *}$ & $0.079 \pm 0.006^{* *}$ & $0.079 \pm 0.005^{* *}$ & $0.080 \pm 0.005^{*}$ & $0.081 \pm 0.005$ & $<0.01$ \\
\hline BRI & $2.89 \pm 1.01^{* *}$ & $3.42 \pm 1.14^{* *}$ & $3.86 \pm 1.20^{* *}$ & $4.22 \pm 1.14^{* *}$ & $4.55 \pm 1.17^{* *}$ & $4.97 \pm 1.33$ & $<0.001$ \\
\hline
\end{tabular}

Data are expressed as mean \pm standard deviation.

$W C$ waist circumference, $B M I$ body mass index, WHR waist-to-hip ratio, WHtR waist-to-height ratio, $B A /$ body adiposity index, $A B S I$ a body shape index, $B R I$ body roundness index, $P_{-}$trend: the median of anthropometric indicators increased significantly with the number of cardiometabolic risk factors. The statistic significances between the highest risk group and other groups are presented by ${ }^{*}$ and ${ }^{* *},{ }^{*} P<0.01 ;{ }^{*} P<0.001$

\section{Discussion}

The present results indicated that WC, BMI, WHR, WHtR and BRI were able to similarly predict high cardiometabolic risk, while BAI or ABSI was not. Moreover, BRI revealed specific predictive ability for elevated TG in the overall population and for elevated SUA in females. The superior predictive capacities and significant associations between BRI and high cardio-metabolic risk were found after adjusted covariates.

Body fat attracted intense attention when it linked with the development of cardiovascular disease and other adverse events $[39,60]$. Generally, the adipose tissue concentrate in the abdomen could produce various compounds of autocrine, paracrine and endocrine activities, which could influence the metabolism and cardiovascular system [61-64]. Obesity-related indicators are non-invasive for predicting body fat mass which will become the simple and practical adiposity markers[65]. The traditional indicators of WC, BMI, WHR, WHtR and BAI were widely recruited to explore the association of adiposity with metabolic disorder, cardiovascular morbidity and diabetes, but the results were unclear due to the delusive relationship with fat mass and distribution [44, 66-68]. Meanwhile, ABSI and BRI were considered as effective indicators for predicting obesity status and risk of metabolic syndrome [51, 69]. A previous study examined the predictive capacity of ABSI and BRI to identify hyperuricemia and to compare the relative strength of association between anthropometric indices and hyperuricemia in rural China [51]. The results found BRI (AUC: 0.641; OR: 1.459) showed more powerful predictive ability for hyperuricemia than BMI (AUC: 0.630; OR: 1.108), while having a similar predictive power as WHtR (AUC: 0.656; OR:1.067) and WC (AUC: 0.658; OR: $1.047)$ in the female group, but not in the male group. However, ABSI had the lowest predictive power for hyperuricemia in both sex categories. Based on the largescale population, the similar results were stably validated in our study and the predictive AUC for hyperuricemia was 0.70 in females. For the predictive capacity and association to cardiometabolic risk factors, BRI could be used as an alternative body index to WHtR, while ABSI could not. Meanwhile, a study with Polish population also showed that BRI had superior ability to predict metabolic syndrome [46]. Generally, BRI possesses a stable ability to predict cardiometabolic risk and metabolic syndrome even in different ethnic groups, and these results were well determined in our study [49]. Moreover, Chang et al. [64]. found that BRI (AUC=0.74), not ABSI was superior measurement compared to BMI, WC and WHtR for determining the presence of left ventricular hypertrophy, especially for eccentric left ventricular hypertrophy [50]. 


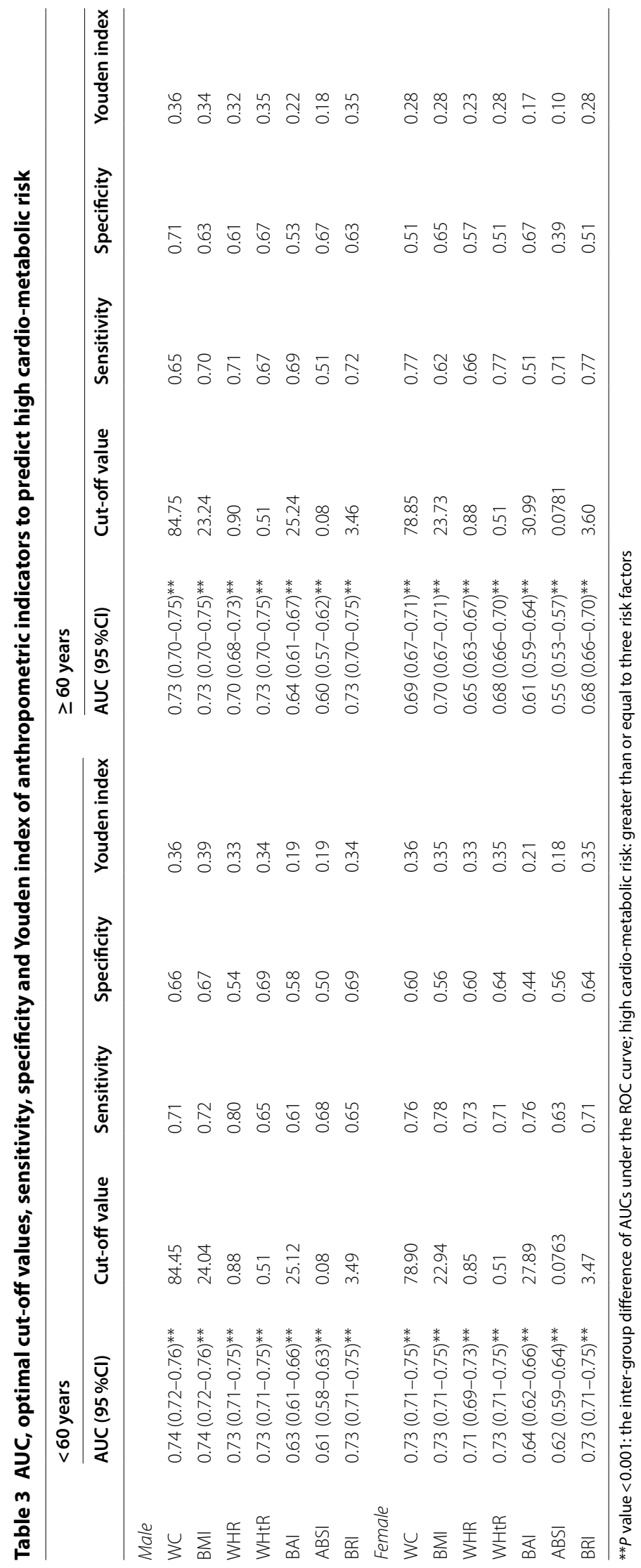


a
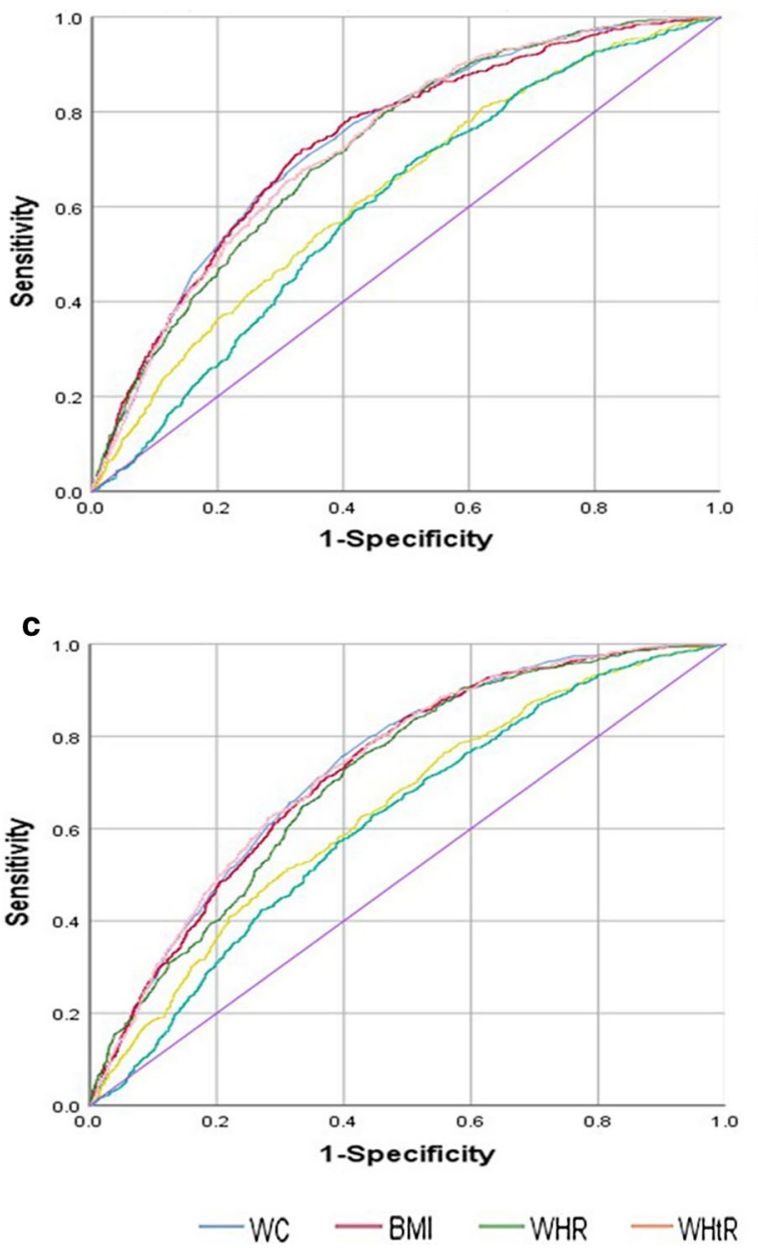

b

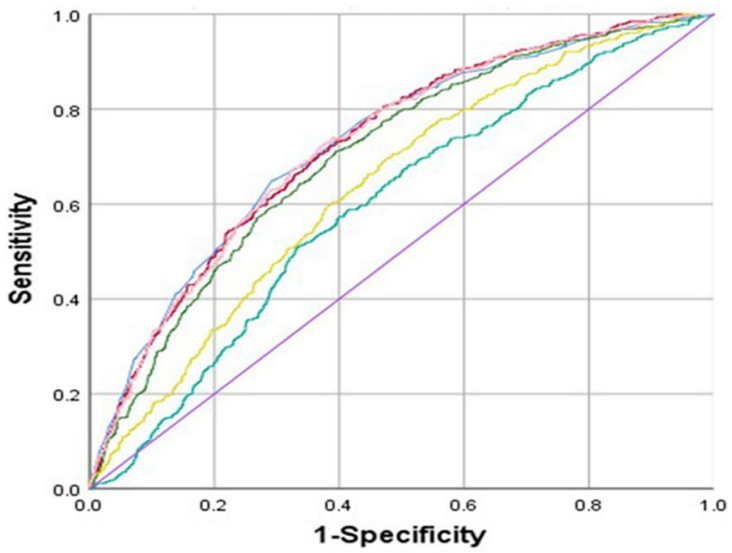

d

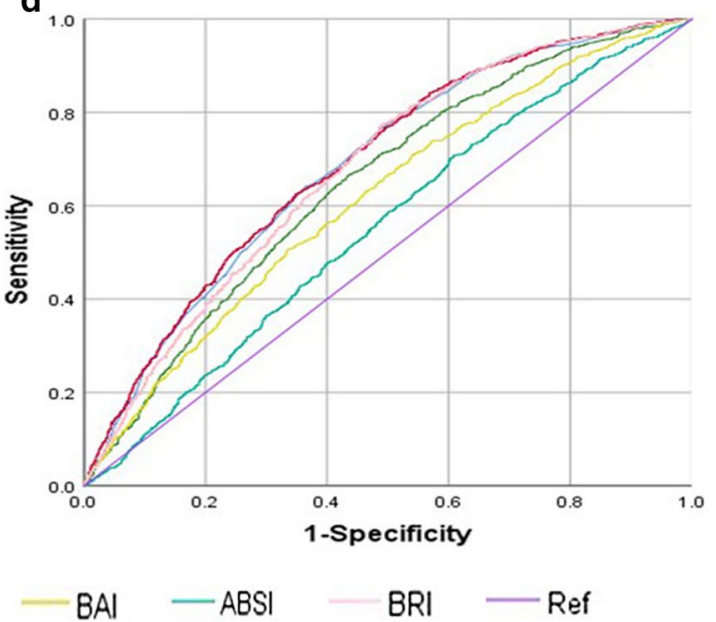

Fig. 2 ROC curves of anthropometric indicators to predict high cardiometabolic risk. a ROC curves in young ( $<60$ years old) group of males. b ROC curves in older ( $\geq 60$ years old) group of males. c ROC curves in young ( $<60$ years old) group of females. $\mathbf{d}$ ROC curves in older ( $\geq 60$ years old) group of females

Obesity was supposed to increase fat accumulation in the cardiac which is associated with ventricular hypertrophy and ventricular systolic dysfunction [70]. Body roundness index (BRI) was developed to predict both body fat and the percentage of visceral adipose tissue using WC in relation to height, which will estimate the shape of the human body figure as an ellipse [4, 43, 71]. BRI was generally used as an adipose indicator for determining the presence of hyperuricemia, arterial stiffness, CVD, diabetes, dyslipidemia, hypertension, and MetSy [7, 46, 49, $51,67,68]$. However, BRI was limited in predicting percentage of fat mass in athletes when compared with bioimpedance analysis or skinfold prediction [72]. Although BRI was not superior to WHtR for determining the presence of MetSy or cardio-metabolic risk, there were no significant differences in predicting MetS, suggesting that BRI was an alternative obesity indicator [43, 73]. The advantages of BRI exceed BMI and WHR were commonly believed to improve the predictive power of body fat and visceral adipose tissue and visceral adipose tissue was associated with MetSy was well known [46, 50,74].

The present study included the large sample size and involved multi-regional population, which avoided the shortcomings of many previous studies. We confirmed BRI was superior indicator for multiple cardio-metabolic risk factors in Chinese adults. Meanwhile, a cross-sectional study also revealed only BRI, not ABSI, can determine the presence of MetSy and insulin resistance (IR) in the overweight and obesity population [75]. Although many advantages were presented, the limitations should 
Table 4 Association of anthropometric indicators with accumulated cardio-metabolic risk factors

\begin{tabular}{|c|c|c|c|c|c|c|c|}
\hline \multirow{2}{*}{$\begin{array}{l}\text { Number of cardio- } \\
\text { metabolic risk } \\
\text { factors }\end{array}$} & \multicolumn{7}{|c|}{ Anthropometric indicators } \\
\hline & WC & BMI & WHR & WHtR & BAI & ABSI & BRI \\
\hline Unadjusted & OR $(95 \% \mathrm{Cl})$ & OR $(95 \% \mathrm{Cl})$ & OR $(95 \% \mathrm{Cl})$ & OR $(95 \% \mathrm{Cl})$ & OR $(95 \% \mathrm{Cl})$ & OR $(95 \% \mathrm{Cl})$ & OR $(95 \% \mathrm{Cl})$ \\
\hline None & Ref & Ref & Ref & Ref & Ref & Ref & Ref \\
\hline 1 & $1.05(1.05-1.06)$ & $1.14(1.13-1.16)$ & $1.37(1.25-1.49)$ & $1.09(1.04-1.13)$ & $1.06(1.05-1.07)$ & $1.08(1.03-1.13)$ & $1.66(1.59-1.73)$ \\
\hline 2 & $1.10(1.09-1.10)$ & $1.29(1.27-1.31)$ & $1.62(1.56-1.69)$ & $1.41(1.18-1.65)$ & $1.12(1.10-1.13)$ & $1.13(1.06-1.20)$ & $2.36(2.26-2.47)$ \\
\hline$\geq 3$ & $1.15(1.14-1.15)$ & $1.45(1.42-1.47)$ & $1.77(1.71-1.83)$ & $1.80(1.74-1.87)$ & $1.17(1.16-1.18)$ & $1.49(1.32-1.66)$ & $3.21(3.05-3.38)$ \\
\hline \multicolumn{8}{|l|}{ Model 1} \\
\hline None & Ref & Ref & Ref & Ref & Ref & Ref & Ref \\
\hline 1 & $1.05(1.04-1.05)$ & $1.15(1.14-1.17)$ & $1.38(1.34-1.41)$ & $1.10(1.00-1.21)$ & $1.07(1.06-1.09)$ & $0.86(0.70-1.02)$ & $1.53(1.46-1.59)$ \\
\hline 2 & $1.09(1.09-1.10)$ & $1.31(1.29-1.33)$ & $1.61(1.57-1.64)$ & $1.39(1.30-1.48)$ & $1.15(1.13-1.16)$ & $1.30(0.90-1.70)$ & $2.17(2.07-2.27)$ \\
\hline$\geq 3$ & $1.14(1.14-1.15)$ & $1.48(1.46-1.51)$ & $1.91(1.88-1.94)$ & $1.56(1.45-1.66)$ & $1.21(1.19-1.23)$ & $1.37(0.82-1.93)$ & $2.94(2.78-3.09)$ \\
\hline \multicolumn{8}{|l|}{ Model 2} \\
\hline None & Ref & Ref & Ref & Ref & Ref & Ref & Ref \\
\hline 1 & $1.06(1.05-1.06)$ & $1.16(1.13-1.18)$ & $1.37(1.31-1.43)$ & $1.20(1.11-1.29)$ & $1.10(1.08-1.12)$ & $0.63(0.23-1.03)$ & $1.67(1.56-1.80)$ \\
\hline 2 & $1.10(1.10-1.11)$ & $1.29(1.26-1.32)$ & $1.60(1.54-1.66)$ & $1.44(1.39-1.50)$ & $1.17(1.15-1.20)$ & $1.02(0.98-1.06)$ & $2.38(2.20-2.57)$ \\
\hline$\geq 3$ & $1.16(1.15-1.17)$ & $1.45(1.41-1.49)$ & $1.79(1.74-1.83)$ & $1.76(1.67-1.85)$ & $1.23(1.21-1.26)$ & $1.08(1.01-1.16)$ & $3.27(3.01-3.55)$ \\
\hline
\end{tabular}

Data are shown as OR ( $95 \% \mathrm{Cls}$ ). The ORs describe the association with increased cardio-metabolic risk factors refer to without cardio-metabolic risk factor group; Model 1: adjusted for age, sex; Model 2: adjusted for model 1 + current smoking, alcohol use, physical activity, alanine transaminase, aspartate transaminase, serum creatinine, blood urea nitrogen

be considered in our study. The numerous obesity indicators were included to predict the cardio-metabolic risk, but the roles of different obesity indicators in presenting fat mass were unique. BMI presented fat mass in the whole body, while WHR was generally regarded as the index in abdominal fat. The obesity indicators were initially conducted to research the associations with cardiovascular disease and mortality in developed countries. We recruited the experienced indicators to predict cardio-metabolic risk in Chinese adults, which may be the main reason why the ABSI failed to predict cardiometabolic risk. In addition, the study was conducted with rural populations residing in southeast China, the unique lifestyle may influence the body shape and metabolic indices. Third, although BRI improved quantification of body shape and provided a more accurate estimate of adipose tissue, the calculation of BRI was so complicated that influenced the clinical application. The longitudinal relationship between BRI and cardio-metabolic risk should be examined in future, meanwhile the multicenters and minority studies are needed to identify the association of obesity indices with more comprehensive metabolic risk factors.

\section{Conclusions}

The present study indicated BRI, WC, BMI, WHR, and WHtR were likely to be optimal indicators for determining the presence of cardio-metabolic risk, especially for high cardio-metabolic risk. BRI was superior and alternative indicator for predicting accumulated cardio-metabolic risk in Chinese adults.

\section{Supplementary Information}

The online version contains supplementary material available at https://doi. org/10.1186/s12872-021-01905-x.

Additional file 1: Table S1. The correlation matrix for multiple anthropometric indicators. WC waist circumference; $H C$ hip circumference; $B M I$ body mass index; WHR waist-to-hip ratio; WHtR waist-to-height ratio; $B A$ I body adiposity index; $A B S /$ a body shape index; $B R I$ body roundness index, ${ }^{*}$ P value $<0.01$. Table S2. Area under curves $(95 \% \mathrm{CI})$ of anthropometric indicators to predict cardio-metabolic risk factors. ${ }^{* *} P$ value $<0.001$, the inter-group difference of AUCs under the ROC curve; WC waist circumference; $H C$ hip circumference; $B M I$ body mass index; WHR waist-to-hip ratio; WHtR waist-to-height ratio; $B A$ I body adiposity index; $A B S /$ a body shape index; $B R I$ body roundness index, $B P$ blood pressure; $F P G$ fasting plasma glucose; TG triglyceride; HDL-C high-density lipoprotein cholesterol; SUA serum uric acid.

Additional file 2: Figure S1. Heatmap of the correlation between anthropometric indicators. (a) The correlation matrix between anthropometric indicators in males; (b) The correlation matrix between anthropometric indicators in females; WC waist circumference; $H C$ hip circumference; BMI body mass index; WHR waist-to-hip ratio; WHtR waist-to-height ratio; BAI body adiposity index; $A B S /$ a body shape index; $B R /$ body roundness index.

Additional file 3: Figure S2. ROC curves of anthropometric indicators to predict multiple cardiometabolic risk factors. (a) ROC curves of anthropometric indicators to predict elevated BP in male; (b) ROC curves of anthropometric indicators to predict elevated FPG in male; (c) ROC curves of anthropometric indicators to predict elevated TG in male; (d) ROC curves of anthropometric indicators to predict reduced $\mathrm{HDL}-\mathrm{C}$ in male; (e) ROC curves of anthropometric indicators to predict elevated SUA in male; (f) ROC curves of anthropometric indicators to predict elevated BP in female; (g) ROC curves of anthropometric indicators to predict elevated FPG in 
female; (h) ROC curves of anthropometric indicators to predict elevated TG in female; (i) ROC curves of anthropometric indicators to predict reduced HDL-C in female; (j) ROC curves of anthropometric indicators to predict elevated SUA in female; WC waist circumference; HC hip circumference; $B M I$ body mass index; WHR waist-to-hip ratio; WHtR waist-to-height ratio; $B A /$ body adiposity index; $A B S /$ a body shape index; $B R I$ body roundness index, BP blood pressure; FPG fasting plasma glucose; TG triglyceride; HDL$C$ high-density lipoprotein cholesterol; SUA serum uric acid.

\section{Abbreviations}

ROC: Receiver operating characteristic curve; AUC: Areas under the curve; CVD: Cardiovascular disease; T2DM: Type 2 diabetes mellitus; EDTA: Ethylene diamine tetraacetic acid; MetSy: Metabolic syndrome; WC: Waist circumference; HC: Hip circumference; BMI: Body mass index; WHR: Waist-to-hip ratio; WHtR: Waist-to-height ratio; BAI: Body adiposity index; ABSI: A body shape index; BRI: Body roundness index; SBP: Systolic blood pressure; DBP: Diastolic blood pressure; FPG: Fasting plasma glucose; TG: Triglyceride; HDL-C: Highdensity lipoprotein cholesterol; LDL-C: Low-density lipoprotein cholesterol; SUA: Serum uric acid; BP: Blood press; ALT: Alanine transaminase; AST: Sspartate transaminase.

\section{Acknowledgements}

Not applicable.

\section{Authors' contributions}

JJX designed the work and drafted the manuscript; LQZ and QW revised the manuscript critically for important intellectual content; YHZ and ZQJ collected and interpreted the data; ZJL created new software used in the work; YMZ were the designer of the manuscript and approved to submit the manuscript finally. All authors read and approved the final manuscript.

\section{Funding}

This work was supported by the grants from National Key Research and Development Program of China (2017YFC0907004) and Hangzhou Science and Technology Project (20171226Y27). The funding agencies had no role in the study design, data collection and analysis, decision to publish or preparation of the manuscript.

\section{Availability of data and materials}

The datasets used and/or analyzed during the current study are available from the corresponding author on reasonable request.

\section{Ethical approval and consent to participate}

Informed and written consent was obtained from all participants, and the protocol was approved by the Zhejiang University ethics committee. The study was conducted according to the ethical guidelines of the Helsinki Declaration and Chinese legislation regarding the protection of personal data was also followed.

\section{Consent for publication \\ Not applicable.}

\section{Competing interests}

The authors declare that they have no competing interests.

\section{Author details}

1 Department of Epidemiology and Biostatistics, School of Public Health, Zhejiang University, Hangzhou 310058, Zhejiang, China. ${ }^{2}$ Putuo District People's Hospital, Zhoushan 316100 , Zhejiang, China. ${ }^{3}$ Department of Respiratory, Sir Run Run Shaw Hospital Affiliated to School of Medicine, Zhejiang University, Hangzhou 310020, Zhejiang, China. ${ }^{4}$ The First Affiliated Hospital, School of Medicine, Zhejiang University, Hangzhou 310058, Zhejiang, China.

Received: 22 August 2020 Accepted: 3 February 2021

Published online: 16 February 2021

\section{References}

1. Saklayen MG. The global epidemic of the metabolic syndrome. Curr Hypertens Rep. 2018;20:2.

2. Akinyemiju T, Moore J, Pisu M, Judd S, Goodman M, Shikany J, et al. A prospective study of obesity, metabolic health, and cancer mortality. Obesity. 2018;26:1.

3. Kachur S, Morera R, De AS, Lavie CJ. Cardiovascular risk in patients with prehypertension and the metabolic syndrome. Curr Hypertens Rep. 2018;20:2.

4. Gu Z, Li D, He H, Wang J, Hu X, Zhang P, et al. Body mass index, waist circumference, and waist-to-height ratio for prediction of multiple metabolic risk factors in Chinese elderly population. Sci Rep. 2018;8:1.

5. Jiang CQ, Xu L, Zhang WS, Jin YL, Zhu F, Cheng KK, et al. Adiposity and mortality in older Chinese: an 11-year follow-up of the Guangzhou Biobank Cohort Study. Sci Rep. 2020;10:1.

6. Li W, Song F, Wang X, Wang D, Lu Z. Relationship between metabolic syndrome and its components and cardiovascular disease in middleaged and elderly Chinese population: a national cross-sectional survey. BMJ Open. 2019;9:8.

7. Liu PJ, Ma F, Lou HP, Zhu YN. Comparison of the ability to identify cardiometabolic risk factors between two new body indices and waist-toheight ratio among Chinese adults with normal BMI and waist circumference. Public Health Nutr. 2017;20:6.

8. Martinelli I, Tomassoni D, Moruzzi M, Roy P, Cifani C, Amenta F, et al. Cardiovascular changes related to metabolic syndrome: evidence in obese zucker rats. Int J Mol Sci. 2020;21:6.

9. Stehouwer CD, Henry RM, Ferreira I. Arterial stiffness in diabetes and the metabolic syndrome: a pathway to cardiovascular disease. Diabetologia. 2008;51:4.

10. Hanley AJ, Karter AJ, Williams K, Festa A, D'Agostino RB Jr, Wagenknecht $L E$, et al. Prediction of type 2 diabetes mellitus with alternative definitions of the metabolic syndrome: the Insulin Resistance Atherosclerosis Study. Circulation. 2005;112:24.

11. Salzano A, D'Assante R, Heaney LM, Monaco F, Rengo G, Valente P, et al. Klinefelter syndrome, insulin resistance, metabolic syndrome, and diabetes: review of literature and clinical perspectives. Endocrine. 2018;61:2.

12. Sanghera DK, Bejar C, Sharma S, Gupta R, Blackett PR. Obesity genetics and cardiometabolic health: potential for risk prediction. Diabetes Obes Metab. 2019;21:5.

13. Iyer A, Fairlie DP, Prins JB, Hammock BD, Brown L. Inflammatory lipid mediators in adipocyte function and obesity. Nat Rev Endocrinol. 2010;6:2.

14. Susic D, Varagic J, Obesity. A perspective from hypertension. Med Clin N Am. 2017;101:1.

15. Ortega FB, Lavie CJ, Blair SN. Obesity and cardiovascular disease. Circ Res. 2016;118:11.

16. Liu LL, Lawrence JM, Davis C, Liese AD, Pettitt DJ, Pihoker C, et al. Prevalence of overweight and obesity in youth with diabetes in USA: the SEARCH for Diabetes in Youth study. Pediatr Diabetes. 2010;11:1.

17. Henderson DC, Vincenzi B, Andrea NV, Ulloa M, Copeland PM. Pathophysiological mechanisms of increased cardiometabolic risk in people with schizophrenia and other severe mental illnesses. Lancet Psychiatry. 2015;2:5.

18. Pope CA, Turner MC, Burnett RT, Jerrett M, Gapstur SM, Diver WR, et al. Relationships between fine particulate air pollution, cardiometabolic disorders, and cardiovascular mortality. Circ Res. 2015;116:1.

19. Haghikia A, Landmesser U. Lipoproteins and cardiovascular redox signaling: role in atherosclerosis and coronary disease. Antioxid Redox Signal. 2018;29:3.

20. Kahn HS, Divers J, Fino NF, Dabelea D, Bell R, Liu LL, et al. Alternative waistto-height ratios associated with risk biomarkers in youth with diabetes: comparative models in the SEARCH for Diabetes in Youth Study. Int J Obes (Lond). 2019;43:10.

21. Kallistratos M, Giannitsi S, Poulimenos L, Miaris N, Koukouzeli A, Khashlok $L A$, et al. Uric acid as a risk factor for cardiovascular disease. A prospective observational study. J Hypertens. 2018;36:3.

22. Borghi C, Rodriguez-Artalejo F, De Backer G, Dallongeville J, Medina J, Nuevo J, et al. Serum uric acid levels are associated with cardiovascular risk score: a post hoc analysis of the EURIKA study. Int J Cardiol. 2018;253:1. 
23. Borghi C, Rosei EA, Bardin T, Dawson J, Dominiczak A, Kielstein JT, et al. Serum uric acid and the risk of cardiovascular and renal disease. J Hypertens. 2015;33:9.

24. Kuwabara M, Hisatome I, Niwa K, Hara S, Roncal-Jimenez CA, Bjornstad $P$, et al. Uric acid is a strong risk marker for developing hypertension from prehypertension: a 5-year Japanese cohort study. Hypertension. 2018;71:1.

25. Li Q, Zhang Y, Ding D, Yang Y, Chen Q, Liu C, et al. Association between serum uric acid and mortality among Chinese patients with coronary artery disease. Cardiology. 2016;134:3.

26. Song M, Li N, Yao Y, Wang K, Yang J, Cui Q, et al. Longitudinal association between serum uric acid levels and multiterritorial atherosclerosis. J Cell Mol Med. 2019:23:8.

27. Wu L, He Y, Jiang B, Liu M, Wang J, Zhang D, et al. Association between serum uric acid level and hypertension in a Chinese elderly rural population. Clin Exp Hypertens. 2017;39:6.

28. Matthias B. Obesity: global epidemiology and pathogenesis. Nat Rev Endocrinol. 2019;12:6

29. Piché M-E, Tchernof A, Després J-P. Obesity phenotypes, diabetes, and cardiovascular diseases. Circ Res. 2020;126:11.

30. Lu J, Wang L, Li M, Xu Y, Ning G. Metabolic syndrome among adults in China: the 2010 China Noncommunicable Disease Surveillance. J Clin Endocrinol Metab. 2016;102:2

31. DR AG, JO T, AM C, MA G, TJ S, et al. The effect of adiposity measured by dual-energy $X$-ray absorptiometry on lung function. Eur Respir J. 2008;32:1.

32. Britton KA, Massaro JM, Murabito JM, Kreger BE, Hoffmann U, Fox CS. Body fat distribution, incident cardiovascular disease, cancer, and allcause mortality. J Am Coll Cardiol. 2013;62:10.

33. Zhao Q, Zhang K, Li Y, Zhen Q, Shi J, Yu Y, et al. Capacity of a body shape index and body roundness index to identify diabetes mellitus in Han Chinese people in Northeast China: a cross-sectional study. Diabetes Med. 2018:35:11.

34. Xing Z, Tang L, Chen J, Pei J, Chen P, Fang Z, et al. Association of predicted lean body mass and fat mass with cardiovascular events in patients with type 2 diabetes mellitus. Cmaj. 2019;191:38.

35. Xing Z, Peng Z, Wang X, Zhu Z, Pei J, Hu X, et al. Waist circumference is associated with major adverse cardiovascular events in male but not female patients with type-2 diabetes mellitus. Cardiovasc Diabetol. 2020;19:1.

36. Aguilar-Morales I, Colin-Ramirez E, Rivera-Mancia S, Vallejo M, VazquezAntona C. Performance of waist-to-height ratio, waist circumference, and body mass index in discriminating cardio-metabolic risk factors in a sample of school-aged Mexican children. Nutrients. 2018;10:12.

37. Elagizi A, Kachur S, Lavie CJ, Carbone S, Pandey A, Ortega FB, et al. An overview and update on obesity and the obesity paradox in cardiovascular diseases. Prog Cardiovasc Dis. 2018;61:2.

38. Hsieh SD, Muto T, Tsuji H, Arase Y, Murase T. Clustering of other metabolic risk factors in subjects with metabolic syndrome. Metabolism. 2010;59:5

39. Jenkins DA, Bowden J, Robinson HA, Sattar N, Loos RJF, Rutter MK, et al. Adiposity-mortality relationships in type 2 diabetes, coronary heart disease, and cancer subgroups in the UK Biobank, and their modification by smoking. Diabetes Care. 2018:41:9.

40. Moliner-Urdiales D, Artero EG, Lee DC, Espana-Romero V, Sui X, Blair SN. Body adiposity index and all-cause and cardiovascular disease mortality in men. Obesity (Silver Spring). 2013;21:9.

41. Myint PK, Kwok CS, Luben RN, Wareham NJ, Khaw KT. Body fat percentage, body mass index and waist-to-hip ratio as predictors of mortality and cardiovascular disease. Heart. 2014;100:20.

42. Quadros TMB, Gordia AP, Andaki ACR, Mendes EL, Mota J, Silva LR. Utility of anthropometric indicators to screen for clustered cardiometabolic risk factors in children and adolescents. J Pediatr Endocrinol Metab. 2019;32:1.

43. Geraci G, Zammuto M, Gaetani R, Mattina A, D'lgnoto F, Geraci C, et al. Relationship of a Body Shape Index and Body Roundness Index with carotid atherosclerosis in arterial hypertension. Nutr Metab Cardiovasc Dis. 2019;29:8

44. Khader Y, Batieha A, Jaddou H, El-Khateeb M, Ajlouni K. The performance of anthropometric measures to predict diabetes mellitus and hypertension among adults in Jordan. BMC Public Health. 2019;19:1.

45. Li G, Wu HK, Wu XW, Cao Z, Tu YC, Ma Y, et al. The feasibility of two anthropometric indices to identify metabolic syndrome, insulin resistance and inflammatory factors in obese and overweight adults. Nutrition. 2019;57:1.

46. Suliga E, Ciesla E, Gluszek-Osuch M, Rogula T, Gluszek S, Koziel D. The usefulness of anthropometric indices to identify the risk of metabolic syndrome. Nutrients. 2019;11:11.

47. Christakoudi S, Tsilidis KK, Muller DC, Freisling H, Weiderpass E, Overvad K, et al. A Body Shape Index (ABSI) achieves better mortality risk stratification than alternative indices of abdominal obesity: results from a large European cohort. Sci Rep. 2020;10:1

48. Li G, Yao T, Wu XW, Cao Z, Tu YC, Ma Y, et al. Novel and traditional anthropometric indices for identifying arterial stiffness in overweight and obese adults. Clin Nutr. 2020;39:3.

49. Rico-Martin S, Calderon-Garcia JF, Sanchez-Rey P, Franco-Antonio C, Martinez Alvarez M, Sanchez Munoz-Torrero JF. Effectiveness of body roundness index in predicting metabolic syndrome: a systematic review and meta-analysis. Obes Rev. 2020;21:7.

50. Chang Y, Guo X, Li T, Li S, Guo J, Sun Y. A Body Shape Index and Body Roundness Index: two new body indices to identify left ventricular hypertrophy among rural populations in Northeast China. Heart Lung Circ. 2016:25:4.

51. Zhang N, Chang Y, Guo X, Chen Y, Ye N, Sun Y. A Body Shape Index and Body Roundness Index: Two new body indices for detecting association between obesity and hyperuricemia in rural area of China. Eur J Intern Med. 2016;29:1

52. Caminha TCS, Ferreira HS, Costa NS, Nakano RP, Assunção ML. Waistto-height ratio is the best anthropometric predictor of hypertension: a population-based study with women from a state of northeast of Brazil. Medicine. 2017;96:2.

53. Fedewa MV, Nickerson BS, Esco MR. Associations of body adiposity index waist circumference, and body mass index in young adults. Clin Nutr. 2018;1:6.

54. Li J, Li J, Wang H, Qi L-W, Zhu Y, Lai M. Tyrosine and glutamine-leucine are metabolic markers of early-stage colorectal cancers. Gastroenterology. 2019;157:1

55. Ruizhi Z, Chengguo L, Chunmei W, Biao Z, Yi L, Feixia P, et al. Natural course of metabolically healthy overweight/obese subjects and the impact of weight change. Nutrients. 2016;8:7.

56. Yimin Z, Dandan Z, Dan Z, Zhenli L, Zhiqiang L, Le F, et al. Susceptibility loci for metabolic syndrome and metabolic components identified in Han Chinese: a multi-stage genome-wide association study. J Cell Mol Med. 2017;21:6.

57. Zheng Q, Lin W, Liu C, Zhou Y, Chen T, Zhang L, et al. Prevalence and epidemiological determinants of metabolically obese but normal-weight in Chinese population. BMC Public Health. 2020;20:1.

58. Alberti KGMM, Zimmet P, Shaw J. Metabolic syndrome-a new worldwide definition. A Consensus Statement from the International Diabetes Federation. Diabetes Med. 2006;23:5.

59. Khichar S, Choudhary S, Singh VB, Tater P, Ujjawal V. Serum uric acid level as a determinant of the metabolic syndrome: a case control study. Diabetes Metab Syndrome Clin Res Rev. 2016;11:1.

60. Kannel WB, Cupples LA, Ramaswami R, Stokes J 3rd, Kreger BE, Higgins M. Regional obesity and risk of cardiovascular disease; the Framingham Study. J Clin Epidemiol. 1991:44:2.

61. Lee JJ, Pedley A, Hoffmann U, Massaro JM, Levy D, Long MT. Visceral and intrahepatic fat are associated with cardiometabolic risk factors above other ectopic fat depots: the Framingham Heart Study. Am J Med. 2018;131:6.

62. Kim Y, Wijndaele K, Lee DC, Sharp SJ, Wareham N, Brage S. Independent and joint associations of grip strength and adiposity with all-cause and cardiovascular disease mortality in 403,199 adults: the UK Biobank study. Am J Clin Nutr. 2017;106:3.

63. Hamer M, Stamatakis E. Metabolically healthy obesity and risk of all-cause and cardiovascular disease mortality. J Clin Endocrinol Metab. 2012;97:7.

64. Moliner-Urdiales D, Artero EG, Lee DC, Espana-Romero V, Sui X, Blair SN. Body adiposity index and all-cause and cardiovascular disease mortality in men. Obesity. 2013;21:9.

65. L CC DVF. E UR, A KJ. Anthropometric indicators as predictors of total body fat and cardiometabolic risk factors in Chilean children at 4, 7 and 10 years of age. Eur J Clin Nutr. 2017;71:4

66. Borel AL, Coumes S, Reche F, Ruckly S, Pépin JL, Tamisier R, et al. Waist, neck circumferences, waist-to-hip ratio: which is the best cardiometabolic 
risk marker in women with severe obesity? The SOON cohort. PLoS One. 2018;13:11.

67. Mohan V, Sandeep S, Deepa M, Gokulakrishnan K, Datta M, Deepa R. A diabetes risk score helps identify metabolic syndrome and cardiovascular risk in Indians - the Chennai Urban Rural Epidemiology Study (CURES38). Diabetes Obes Metab. 2007;9:3.

68. Rådholm K, Chalmers J, Ohkuma T, Peters S, Poulter N, Hamet P, et al. Use of the waist-to-height ratio to predict cardiovascular risk in patients with diabetes: results from the ADVANCE-ON study. Diabetes Obes Metab. 2018;20:8.

69. Bertoli S, Leone A, Krakauer NY, Bedogni G, Battezzati A. Association of Body Shape Index (ABSI) with cardio-metabolic risk factors: a crosssectional study of 6081 Caucasian adults. Plos One. 2017;12:9.

70. $B P L, A B D C, C R, A E G B$. Effect of obesity on left ventricular mass and systolic function in patients with asymptomatic aortic stenosis (a Simvastatin Ezetimibe in Aortic Stenosis (SEAS) substudy). Am J Cardiol. 2010;105:10.

71. Ibiza A-M, Eloisa C-R, Susana R-M, Maite V, Clara V-A. Performance of waist-to-height ratio, waist circumference, and Body Mass Index in discriminating cardio-metabolic risk factors in a sample of school-aged Mexican children. Nutrients. 2018;10:12.

72. A SD, M SA. N MC, P MJ, S MC, M TD, et al. Utility of novel body indices in predicting fat mass in elite athletes. Nutrition. 2015;31:7-8.

73. Bertoli S, Leone A, Krakauer NY, Bedogni G, Vanzulli A, Redaelli Vl, et al. Association of Body Shape Index (ABSI) with cardio-metabolic risk factors: a cross-sectional study of 6081 Caucasian adults. PLoS One. 2017;12:9.

74. Jing D, Si-Si W, Xi C, et al. Optimal cut-off point of waist to height ratio in Beijing and its association with clusters of metabolic risk factors. Curr Med Sci. 2019;39:2.

75. Gang L, Hui-Kun W, Xiao-Wei W, Zhe C, Yuan-Chao T, Yi M, et al. The feasibility of two anthropometric indices to identify metabolic syndrome, insulin resistance and inflammatory factors in obese and overweight adults. Nutrition. 2019:57:1.

\section{Publisher's note}

Springer Nature remains neutral with regard to jurisdictional claims in published maps and institutional affiliations.
Ready to submit your research? Choose BMC and benefit from:

- fast, convenient online submission

- thorough peer review by experienced researchers in your field

- rapid publication on acceptance

- support for research data, including large and complex data types

- gold Open Access which fosters wider collaboration and increased citations

- maximum visibility for your research: over $100 \mathrm{M}$ website views per year

At BMC, research is always in progress.

Learn more biomedcentral.com/submissions 